\title{
Severe dysphagia requiring gastrostomy following cervical spine fracture fixation
}

\author{
Joanelle A Bailey, ${ }^{1}$ Robert F Lavery, ${ }^{1}$ John M Adams, ${ }^{2}$ Andrew S Livingston, ${ }^{1}$ \\ Lou DiFazio, ${ }^{2}$ David H Livingston'
}

${ }^{1}$ Rutgers University-New Jersey Medical School, Newark, New Jersey, USA

${ }^{2}$ Divisions of Trauma and Surgical Critical Care, Department of Surgery at Morristown Memorial Hospital, Morristown, New Jersey, USA

\section{Correspondence to}

Dr David H Livingston; Rutgers University - New Jersey Medical School, University Hospital M234, 150 Bergen Street, Newark, NJ 07103 USA; livingst@rutgers.edu

Presented in part as a Poster Presentation at the 2016 Annual Southeastern Surgical Congress, February 20-23, 2016, Atlanta, Georgia, USA.

Received 8 March 2016 Revised 6 May 2016 Accepted 11 May 2016

\footnotetext{
To cite: Bailey $\mathrm{JA}$,

Lavery RF, Adams JM, et al. Trauma Surg Acute Care Open Published Online First: [please include Day Month Year] doi:10.1136/tsaco2016-000001
}

\author{
ABSTRACT \\ Background The incidence of severe dysphagia \\ requiring gastrostomy tube (GT) placement following \\ operative fixation of traumatic cervical spine fractures is \\ unknown. Risk factors for severe dysphagia are not well \\ identified and GT placement is often delayed due to the \\ belief that it will resolve quickly. We hypothesized that \\ patient and clinical factors could be used to predict \\ severe dysphagia requiring GT placement in this \\ population.
}

Methods A retrospective multicenter review of all adult patients requiring operative fixation of cervical spine fractures was performed. Data on demographics, injury severity score, presence of spinal cord injury, operative approach, presence of severe traumatic brain injury, and the need and timing of tracheostomy and GT were collected. The timing, number and results of formal speech, and language pathology examinations were also recorded.

Results 243 patients underwent cervical spine fixation for traumatic fractures, of which $72(30 \%)$ required GT placement. Patients requiring gastrostomy were significantly older, 54 versus 45 years $(p=0.002)$, and had higher injury severity scores at 24 versus 18 $(p<0.0001)$. Tracheostomy was strongly associated with severe dysphagia; GT was required in $83 \%$ of patients who underwent tracheostomy versus $5 \%$ of those who did not require tracheostomy. $50 \%$ of patients underwent tracheostomy and GT on the same day after injury, with the remaining patients having an average of 9 days delay between procedures. The need for gastrostomy placement was also higher in patients undergoing combined operative approach versus anterior or posterior approach alone $(p=0.02)$. There were no GT-related complications.

Conclusions Severe dysphagia requiring GT placement occurs commonly $(30 \%)$ in patients who undergo operative fixation of cervical spine fractures. Gastrostomy placement was delayed in 50\%. Tracheostomy was strongly associated with the need for GT placement. Earlier GT placement, especially in patients requiring tracheostomy, would improve patient care and disposition.

\section{INTRODUCTION}

Dysphagia severe enough to require gastrostomy tube (GT) placement is thought to be an uncommon event following operative management of cervical spine pathology, but the incidence is unknown. Most literature analyzing dysphagia following cervical spine operations has focused primarily on patients with elective discectomy and has generally excluded trauma patients. The published incidence of dysphagia following elective operative fixation varies widely and has been reported between $2 \%$ and $71 \% .^{1-5}$ While the anterior operative approach has commonly been thought to be associated with the development of dysphagia, assessment of various operative approaches by Smith-Hammond et $a l^{6}$ did not find that any specific operative approach correlated with an increased risk of dysphagia. Furthermore, literature examining this topic has used variable definitions and determinants for dysphagia and even the most symptomatic of patients in these studies did not require gastrostomy placement. ${ }^{578}$ Another limitation of these studies includes reliance on selfreported symptoms rather than a standardized objective assessment of symptoms. ${ }^{7}$ Potential mechanisms of dysphagia are numerous, including soft tissue edema, stretch injury of nerves, and retraction of structures involved in the swallowing mechanism. 7910 Most importantly, while these patients may experience subjective dysphagia, it rarely requires GT placement and almost always resolves spontaneously within a year or less. ${ }^{11}$

Our experience in the care of patients with traumatic cervical spine pathology contrasts sharply with that of the elective literature with many of our patients requiring GT placement. We postulated that the incidence of severe dysphagia requiring surgical feeding access is significantly higher in patients with traumatic cervical spine fractures requiring operative fixation. Additionally, experience with elective patients, for whom dysphagia is a self-limited problem, influences the perceptions of trauma and spine surgeons resulting in significant delays in feeding access placement. We also hypothesized that identifiable patient and clinical factors could be used to predict severe dysphagia, which could help providers select patients for early gastrostomy placement.

The goals of this study were to determine the incidence of severe dysphagia following operative intervention for traumatic cervical spine injury. We also hoped to identify factors that influenced the development of severe dysphagia and would be predictive of a need for GT placement. Additionally, we attempted to evaluate the efficacy and utility of objective speech and swallow evaluations in the management of these patients and whether repetition of these evaluations impacted the necessity of surgical feeding access. 


\section{METHODS}

All adult patients with traumatic cervical spine pathology who underwent operative fixation procedures by the Neurosurgery or Orthopedic Spine services at The New Jersey Trauma Center in Newark, New Jersey and at Morristown Memorial Hospital, Morristown, New Jersey between 2000 and 2014 were reviewed retrospectively. This study was approved by the Institutional Review Boards of Rutgers University-New Jersey Medical School and Morristown Memorial Hospital.

Data collected included demographics, injury severity scores (ISS), presence of severe traumatic brain injury (TBI) as defined by abbreviated injury score (AIS) $\geq 3$, presence of spinal cord injury (SCI), operative approach (anterior, posterior, or combined), and the need and timing of tracheostomy and GT placements. The timing, number, and outcomes of speech and language pathology therapist administered swallow evaluations (speech and language, SLP) were noted.

In patients who underwent swallow evaluations, dysphagia was objectively defined by failing that study. In patients who did not undergo formal SLP evaluations, the presence of dysphagia was based on the clinical impression and judgment of the treating physicians.

Statistical analysis was conducted using parametric and nonparametric studies, with $\chi^{2}$ and unpaired t-test.

\section{RESULTS}

A total of 243 patients underwent operative cervical spine fixation for traumatic fractures. The mean age of this group was 48 years with a range of $18-99 ; 175(72 \%)$ of these patients were male. Of the total patients, $72(30 \%)$ required gastrostomy; 4 were open and 68 were percutaneous endoscopic gastrostomy; there were no complications associated with GT placement. The demographic details are summarized in table 1 .

Patients requiring gastrostomy placement were older with a mean age of 54 versus 45 years for those not requiring gastrostomy $(\mathrm{p}=0.0021)$. These patients were also more severely injured, with ISS of 24 versus $18(\mathrm{p}<0.0001)$ for those who did not require gastrostomy. Eighty-nine (37\%) patients had significant TBI (AIS $\geq 3$ ), with only 30 (34\%) requiring GT placement. Approximately half of the patients (49\%) had a SCI; of these patients, $43(36 \%)$ required GT placement, but the majority did not. Neither severe TBI nor SCI was associated with an increased need for GT in this population. These data are presented in table 2.

Overall, 72 (30\%) patients required gastrostomy placement, and 77 (32\%) required tracheostomy; significant overlap of these patients existed, with many of them requiring both GT and tracheostomy. Of those requiring tracheostomy, 64 (83\%) had severe dysphagia necessitating GT placement. Timing of the procedures was available for 57 (89\%) of the patients requiring tracheostomy and GT placement. Twenty-nine patients (51\%) underwent both procedures on the same day, whereas 28 (49\%)

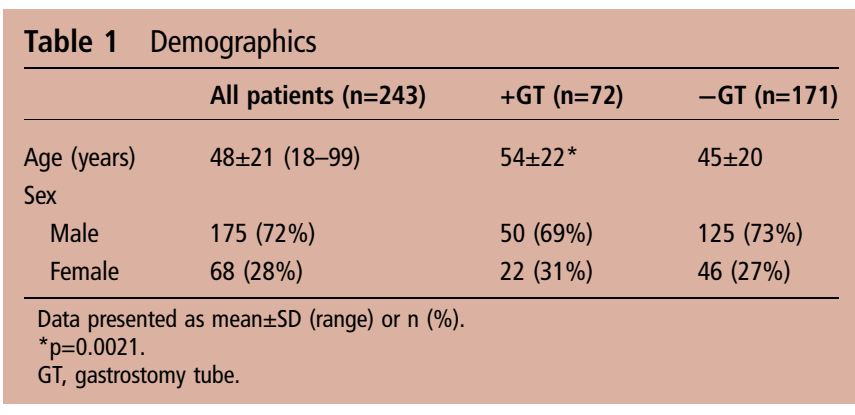

had these procedures performed on different days, with an average delay between procedures of 9 days. Gastrostomy was not required in $13(17 \%)$ patients who underwent tracheostomy; half of these patients were under 70 years of age and none had significant TBI.

Patients who required anterior and posterior combined operative approaches had a higher rate of severe dysphagia requiring GT, compared to anterior or posterior approach alone $(p=0.02)$, but there was a significant rate of dysphagia in all groups. Of note, $31 \%$ of patients undergoing a solely posterior approach required a GT, which was higher than those undergoing an anterior approach.

Ninety-nine $(38 \%)$ patients underwent formal swallow evaluations (SLP) by certified speech and language pathology therapists. Of those who underwent evaluation, 43 (46\%) eventually required gastrostomy and $50(54 \%)$ did not. The average number of evaluations was three for those who did inevitably require GT placement and two for those who did not. The mean time from the first SLP evaluation to GT placement was 7 days.

\section{DISCUSSION}

The incidence of severe dysphagia necessitating surgical gastrostomy placement occurs in about one in three of all patients with traumatic cervical spine fractures who require operative fixation; this increases to over $80 \%$ in those patients who necessitate tracheostomy. Even in those patients who did not require a tracheostomy, 5\% ultimately required feeding access. The rate of severe dysphagia in this population far exceeds the incidence previously reported in patients undergoing elective cervical spine surgery. ${ }^{1-5}$

In half of the patients who required tracheostomy and gastrostomy, the GT was delayed, an average of 9 days. While the reason for this delay is difficult to fully ascertain from this chart review, we strongly believe that it is a misperception in the true incidence of severe dysphagia in this population. Patients waiting for GT placement would have required alternate enteral feeding access via nasogastric tube (NGT). Given the retrospective nature of this study, it was not possible to capture data such as days with NGT in place or frequency of inadvertent pulling of the NGT and requiring subsequent reinsertion. These common events increase patient discomfort, require additional radiologic studies to confirm placement and replacement, and affect caloric intake from interruption of nutritional support. All of these factors are additional motives for earlier GT placement. Given the strong association between tracheostomy and gastrostomy, we advocate a change in management and recommend performing these procedures on the same day.

The anterior surgical approach has most commonly been implicated with clinically significant dysphagia following elective cervical spine surgery. ${ }^{1-5} 7^{9-12}$ Our data confirms the findings of Smith-Hammond et $a l^{6}$ showing that all approaches were associated with significant rates of severe dysphagia. Not surprisingly, patients who required both approaches had the highest incidence of dysphagia.

The definitive role of SLP evaluations to aid in the diagnosis and management of dysphagia in this population is unfortunately difficult to assess from the data. About half of the patients who underwent SLP did not require GT. However, there was a 7-day delay from the first to second SLP with an average of three SLP evaluations for those patients who eventually required a GT. Given the strong association between the need for tracheostomy and GT placement we would recommend that SLP evaluations be limited to those patients not requiring tracheostomy. 
Table 2 Factors influencing the need for gastrostomy

\begin{tabular}{|c|c|c|c|c|}
\hline & All patients & $+\mathrm{GT}$ & $-\mathrm{GT}$ & $\mathrm{p}$ Value \\
\hline Total patients & 243 & $72(30 \%)$ & $171(70 \%)$ & \\
\hline PEG & & $68(94 \%)$ & & \\
\hline Open & & $4(6 \%)$ & & \\
\hline ISS & $20 \pm 10$ & $24 \pm 10$ & $18 \pm 10$ & $<0.0001$ \\
\hline Severe TBI & $89(37 \%)$ & $30(34 \%)$ & $59(66 \%)$ & 0.29 \\
\hline $\mathrm{SCl}$ & $119(49 \%)$ & $43(36 \%)$ & $76(64 \%)$ & 0.36 \\
\hline Tracheostomy & 77 (32\%) & $64(83 \%)$ & $13(17 \%)$ & $<0.0001$ \\
\hline Same day & & $29(51 \%)$ & & \\
\hline Different day & & $28(49 \%)$ & & \\
\hline No tracheostomy & $166(68 \%)$ & $8(5 \%)$ & 158 (95\%) & \\
\hline \multicolumn{5}{|l|}{ Operative approach $(n=231)$} \\
\hline Anterior & $95(41 \%)$ & $22(23 \%)$ & $73(77 \%)$ & \multirow{5}{*}{$\begin{array}{l}0.02 \text { combined versus } \\
\text { posterior or anterior }\end{array}$} \\
\hline Posterior & $107(46 \%)$ & $33(31 \%)$ & $74(69 \%)$ & \\
\hline Both & $29(13 \%)$ & $14(48 \%)$ & $15(52 \%)$ & \\
\hline SLP & $93(38 \%)$ & $43(46 \%)$ & $50(54 \%)$ & \\
\hline Number of evaluations & & 3 & 2 & \\
\hline
\end{tabular}

It also seemed that repeated SLP evaluations did not impact greatly on whether patients will require gastrostomy placement and patients who required more than two evaluations always underwent GT placement. Therefore, after two failed SLP evaluations patients should undergo gastrostomy placement. Even if the patient fails a single SLP evaluation, strong consideration should be given to GT placement versus attempting another SLP study, which might potentially prolong hospitalization.

The recommendations made from these data are the risks and benefits of all procedures and courses of therapy. The prolonged use of an NGT and the delays involved in patient care are real, but are often not considered compared to more invasive procedures. While no patient sustained GT-related complications (such as misplacement, bleeding, and infection), these are real and the morbidity associated with the procedure needs to be taken into account. ${ }^{13}$ However, given the high incidence of significant dysphagia in this patient population, early use of surgical GT placement should be strongly considered.

The strengths of these data include that the population consists of only operative trauma patients, which have been underrepresented in other series. ${ }^{3} 679$ A limitation of this retrospective study is that we did not rigorously define dysphagia. Unfortunately not all patients underwent objective evaluation of dysphagia as measured by the speech and language pathologists. As only $38 \%$ of patients were objectively evaluated by speech and language pathology, assumptions on the remaining $62 \%$ can only be extrapolated. In the group that did undergo SLP evaluation, $25 \%$ passed on the first evaluation with just under half never passing by the third attempt. Thus, we believe that over half of the patients had objectively documented severe dysphagia. While the incidence of dysphagia did not appear to change over the course of the study, it is possible that operative techniques (eg, lower profile plates) may have changed or have varied by specialty (neurosurgery vs orthopedics) that could influence dysphagia. Age and presence of neurological injury (TBI or SCI) may confound, but do not obviate the conclusions.

\section{CONCLUSION}

In conclusion, patients with traumatic cervical spine fractures requiring operative fixation have a high incidence of severe dysphagia necessitating GT placement. This dysphagia is clinically significant and requires operative intervention. Tracheostomy is associated with an almost universal need for GT placement. Therefore, patients who require tracheostomy should have gastrostomy placed simultaneously in order to streamline patient care and avoid the morbidity associated with delayed placement and multiple procedures. The role of objective swallow evaluations to aid in the management of dysphagia in this population remains difficult to assess. SLP evaluations should be performed only in patients not undergoing tracheostomy, and more than one failure should be indication for GT, as further evaluations did not change outcome but did delay gastrostomy placement.

Contributors DHL and RFL analyzed study design, data analysis, manuscript preparation and editing, and final approval. JAB, ASL, LD, and JMA carried out data acquisition and analysis, manuscript preparation, and editing and final approval.

Competing interests None declared.

Provenance and peer review Not commissioned; externally peer reviewed.

Open Access This is an Open Access article distributed in accordance with the Creative Commons Attribution Non Commercial (CC BY-NC 4.0) license, which permits others to distribute, remix, adapt, build upon this work non-commercially, and license their derivative works on different terms, provided the original work is properly cited and the use is non-commercial. See: http://creativecommons.org/ licenses/by-nc/4.0/

\section{REFERENCES}

1 Lee MJ, Bazaz R, Furey CG, et al. Risk factors for dysphagia after anterior cervical spine surgery: a two-year prospective cohort study. Spine J 2007;7:141-7.

2 Lee JY, Lim MR, Albert TJ. Dysphagia after anterior cervical spine surgery: pathophysiology, incidence, and prevention. Cervical Spine Res Soc 2005. http:// www.csis.org/wp-content/uploads/2014/06/dysphagia-outcome-study.pdf (accessed May 28, 2016)

3 Joaquim AF, Murar J, Savage JW, et al. Dysphagia after anterior cervical spine surgery: a systematic review of potential preventative measures. Spine J 2014; 14:2246-60.

4 Rihn JA, Kane J, Albert TJ, et al. What is the incidence and severity of dysphagia after anterior cervical surgery? Clin Orthop Relat Res 2011;469:658-65.

5 Riley LH III, Vaccaro AR, Dettori JR, et al. Postoperative dysphagia in anterior cervical spine surgery. Spine 2010;35(9 Suppl):S76-85.

6 Smith-Hammond CA, New KC, Pietrobon R, et al. Prospective analysis of incidence and risk factors of dysphagia in spine surgery patients: comparison of anterior cervical, posterior cervical, and lumbar procedures. Spine 2004;29:1441-6.

7 Anderson KK, Arnold PM. Oropharyngeal dysphagia after anterior cervical spine surgery: a review. Global Spine J 2013;3:273-86. 
8 Chen CJ, Saulle D, Fu KM, et al. Dysphagia following combined anterior-posterio cervical spine surgeries. J Neurosurg Spine 2013;19:279-87.

9 Cho SK, Lu Y, Lee DH. Dysphagia following anterior cervical spinal surgery: a systematic review. Bone Joint J 2013;95-B:868-73.

10 Khaki F, Zusman NL, Nemecek AN, et al. Postoperative prevertebral soft tissue swelling does not affect the development of chronic dysphagia following anterior cervical spine surgery. Spine 2013;38:E528-32.
11 Kalb S, Reis MT, Cowperthwaite MC, et al. Dysphagia after anterior cervical spine surgery: incidence and risk factors. World Neurosurg 2012;77:183-7.

12 Wu B, Song F, Zhu S. Reasons of dysphagia after operation of anterior cervical decompression and fusion. J Spinal Disord Tech. Published Online First: Oct 10, 2014.

13 Yuruker S, Koca B, Karabicak I, et al. Percutaneous endoscopic gastrostomy: technical problems, complications, and management. Indian I Surg 2015;77(Suppl 3):1159-64. 